\title{
Using Compact LoRa Devices for In-Building Communications
}

\author{
Felisberto Pereira ${ }^{1,3}$, Sérgio I. Lopes ${ }^{1,2, *}$, José M. N. Vieira ${ }^{1,3}$, \\ Nuno B. Carvalho ${ }^{1,3}$ and António Curado ${ }^{2,4}$ \\ ${ }^{1}$ Instituto de Telecomunicações, Campus Universitário de Santiago, Aveiro, Portugal \\ ${ }^{2}$ Instituto Politécnico de Viana do Castelo, Viana do Castelo, Portugal \\ ${ }^{3}$ DETI, University of Aveiro, Campus Universitário de Santiago, Portugal \\ ${ }^{4}$ CONSTRUCT LFC, Faculty of Engineering (FEUP), University of Porto, Portugal \\ sileestg.ipvc.pt
}

\begin{abstract}
In [1], Buckman Et. al. define a Smart Building as the harmonious integration of intelligent systems, control mechanisms, architecture and construction materials to operate as an entire building system, with adaptability at its core to enable continuous building improvement in terms of energy efficiency, longevity, comfort, and satisfaction. The development of intelligent devices for these Smart Buildings is many times compromised by its form and size. This work explores the limitations due to antenna design restrictions, that are normally responsible for design constraints, power issues, used materials and component placement (e.g. sensors, batteries, etc). The work focuses on the importance of the antenna design, considering many aspects as the presence of surrounding materials, the device's encapsulation and the proximity between the antenna and other electronic circuits. These aspects are especially important in devices designed for Smart Building applications where form, size, range, and power consumption impose severe restrictions.

The proposed design features a reduced size embedded antenna and an ultra-lowpower microcontroller to interface several sensors and actuators. The results obtained have shown that the proposed device can be used for communications, in line-of-sight for up to $4.2 \mathrm{~km}$, in urban environments for up to $1.2 \mathrm{~km}$ and for inbuilding communications for up to $152 \mathrm{~m}$, without compromising the low-power features that LoRa supports. This technology allows a node capable of measure $\mathrm{CO}_{2}$, Temperature, Humidity, and Air Pressure to be powered by a CR2477 coin cell (that has a power capacity of $1000 \mathrm{mAh}$ ) over a period of 3 years and 7 months, approximately.

This demo explores the concept previously introduced in the context of a Smart Building, not only for in-building communications but also for communications between buildings by means of a set of compact LoRa devices in operation.
\end{abstract}

Keywords: Green Communications $\cdot$ LoRa $\cdot$ Smart Buildings.

\section{References}

1. A.H. Buckman, M. Mayfield, Stephen B.M. Beck, "What is a Smart Building?", Smart and Sustainable Built Environment, 2014, Vol. 3 Issue: 2, pp.92-109. https://doi.org/10.1108/SASBE-01-2014 